\title{
Estimating and forecasting the precipitable water vapor from GOES satellite data at high altitude sites
}

\author{
Julio C. Marín ${ }^{1}$, Diana Pozo ${ }^{1}$, and Michel Curé ${ }^{2}$ \\ ${ }^{1}$ Departamento de Meteorología, Universidad de Valparaíso, Casilla 5030, Chile \\ e-mail: julio.marin@meteo.uv.cl \\ 2 Instituto de Física y Astronomía, Universidad de Valparaíso, Casilla 5030, Chile \\ e-mail: michel. cure@uv.cl
}

Received 24 June 2014 / Accepted 16 October 2014

ABSTRACT

\begin{abstract}
In this work, we describe a method to estimate the precipitable water vapor (PWV) from Geostationary Observational Environmental Satellite (GOES) data at high altitude sites. The method was applied at Atacama Pathfinder Experiment (APEX) and Cerro Toco sites, located above $5000 \mathrm{~m}$ altitude in the Chajnantor plateau, in the north of Chile. It was validated using GOES-12 satellite data over the range $0-1.2 \mathrm{~mm}$ since submillimeter/millimeter astronomical observations are only useful within this PWV range. The PWV estimated from GOES and the Final Analyses (FNL) at APEX for 2007 and 2009 show root mean square error values of $0.23 \mathrm{~mm}$ and $0.36 \mathrm{~mm}$ over the ranges $0-0.4 \mathrm{~mm}$ and $0.4-1.2 \mathrm{~mm}$, respectively. However, absolute relative errors of $51 \%$ and $33 \%$ were shown over these PWV ranges, respectively. We recommend using high-resolution thermodynamic profiles from the Global Forecast System (GFS) model to estimate the PWV from GOES data since they are available every three hours and at an earlier time than the FNL data. The estimated PWV from GOES/GFS agrees better with the observed PWV at both sites during night time. The largest errors are shown during daytime. Short-term PWV forecasts were implemented at both sites, applying a simple persistence method to the PWV estimated from GOES/GFS. The $12 \mathrm{~h}$ and $24 \mathrm{~h}$ PWV forecasts evaluated from August to October 2009 indicates that $25 \%$ of them show a very good agreement with observations whereas $50 \%$ of them show reasonably good agreement with observations. Transmission uncertainties calculated for PWV estimations and forecasts over the studied sites are larger over the range $0-0.4 \mathrm{~mm}$ than over the range $0.4-1.2 \mathrm{~mm}$. Thus, the method can be used over the latter interval with more confidence.
\end{abstract}

Key words. atmospheric effects - methods: numerical - methods: data analysis

\section{Introduction}

The water vapor in the atmosphere absorbs and emits infrared (IR) radiation in a broad band of the electromagnetic spectrum, which represents a great challenge for submillimeter/millimeter observations at the Earth surface. A number of site testing studies have been conducted to find good places for submillimeter/millimeter astronomy (Peterson et al. 2003; Yang et al. 2010; Radford et al. 2011; Tremblin et al. 2011, 2012), and they have identified Mauna Kea in Hawaii, the Chajnantor plateau in the north of Chile, and some regions in the south pole as very good sites.

A number of astronomical observatories are installed in the north of Chile (e.g. the Atacama Pathfinder Experiment (APEX), the Atacama Large Millimeter Array (ALMA) and some instrumentation in Paranal) that operate at IR bands. The quality of their observations are largely affected by the atmospheric humidity. Therefore, it is very important for them to know accurately the amount of water vapor in the atmosphere in order to have a better observation planning and to reduce the costs of their scientific operations.

The precipitable water vapor (PWV), which is equivalent to the depth of liquid water that would result if all the water vapor in the atmospheric column is condensed and precipitated, is used to diagnose the atmospheric humidity over a specific location. The PWV can be estimated directly or indirectly using different methods. Radiosondes can be used to directly estimate the PWV (Motell et al. 2002; Li et al. 2003), although a number of studies have mentioned the humidity measurement uncertainties, such as solar heating of the relative humidity (RH) sensor and lag-time response of the instrument at low temperatures. For that reason, several corrections have been derived to remove the mean bias error as a function of different parameters (Miloshevich et al. 2004, 2006, 2009; Vömel et al. 2007; Cady-Pereira et al. 2008). Indirect methods estimate the PWV from the atmospheric transmittances and emissions at infrared or radio wavelengths. These include ground-based radiometers (Peterson et al. 2003; Otálora et al. 2010; Radford 2011), radiometers onboard satellites (Soden \& Bretherton 1993; Motell et al. 2002; Tobin et al. 2006), or global positioning system (GPS) data (Duan et al. 1996; Li et al. 2003; Wu et al. 2003).

A number of studies have been carried out to analyze the temporal and spatial behavior of PWV over astronomical observatories using remote-sensing data. Kurlandczyk \& Sarazin (2007) used Medium Resolution Imaging Spectrometer (MERIS) data onboard the polar Environmental Satellite (Envisat) to estimate the cloud cover and PWV to select the best sites for the installation of the European Extremely Large Telescope (E-ELT). Yang et al. (2010) and Tremblin et al. (2011, 2012) used polar satellite data for comparative studies among different sites to conduct submillimeter/millimeter astronomy. Nevertheless, the main drawback of estimating the PWV from 


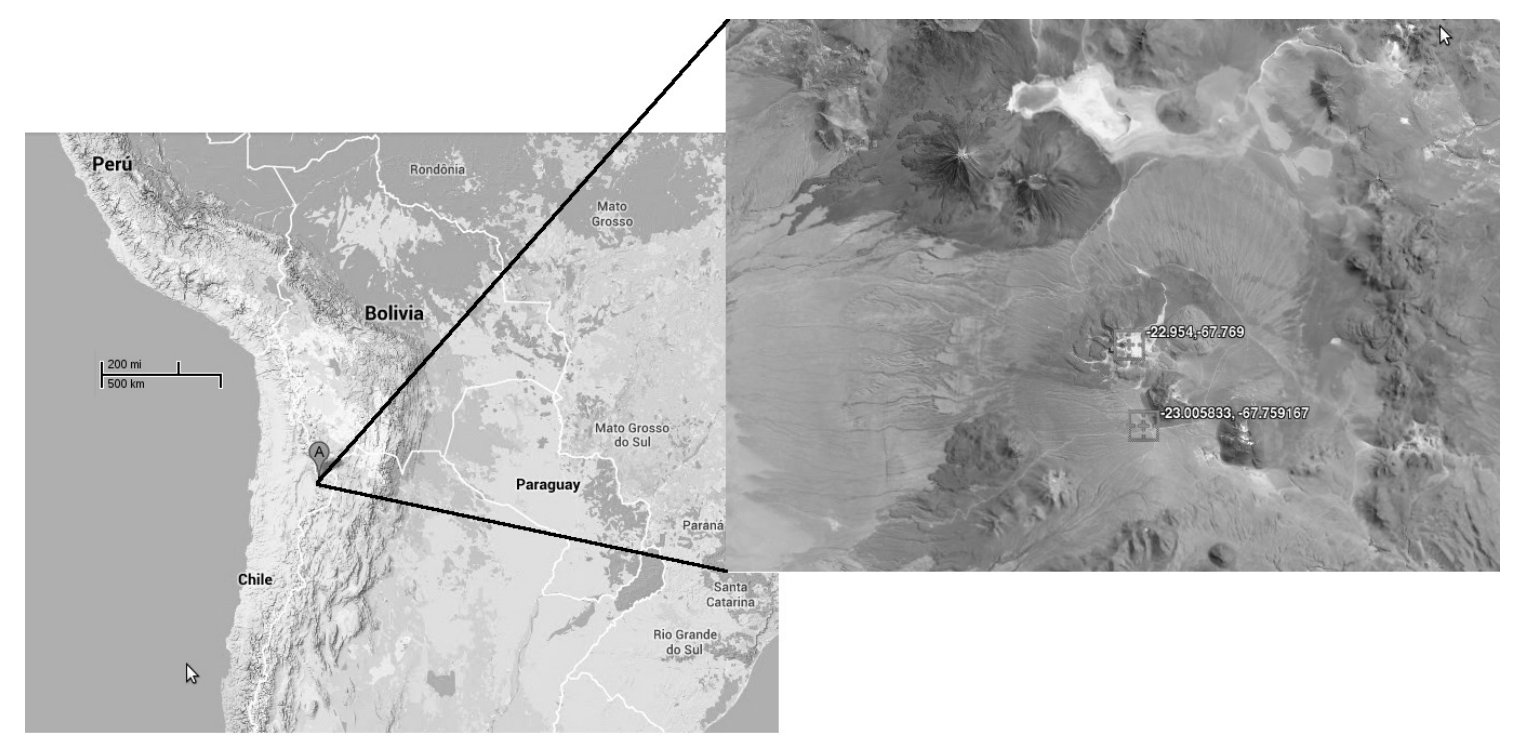

Fig. 1. Location of APEX and RHUBC-II sites in the Atacama Desert in the north of Chile (square marks in right panel).

polar satellites is their low temporal resolution since they pass every two or three days over a specific spot on Earth at almost the same hour of the day.

Erasmus (2002), Erasmus \& Van Staden (2001) used GOES-8 (Geostationary Operational Environmental Satellite) data to estimate the PWV and the cloud cover at astronomical sites. Geostationary satellites have the benefit of providing data at a higher temporal resolution (15-30 min). The Erasmus and van Staden's methodology is based on an equation from Soden \& Bretherton (1993) that relates the GOES-7 satellite brightness temperature with the upper tropospheric humidity (see Eq. (5) in Sect. 2.3.2). To obtain the PWV, a water vapor profile is needed. Their methodology used monthly means profiles from the Antofagasta rawinsonde at 00 and 12 UTC to provide humidity information every three hours.

This paper presents a methodology to estimate the PWV at high altitude sites from GOES-12 satellite data. Its main value is that it is an easy and straightforward way to estimate the PWV over a specific site where no other means to obtain the PWV are available. The methodology is based on Erasmus \& van Staden (2001), but differs in two main respects; first, a new cloud-clearance algorithm is implemented to obtain clear-sky brightness temperatures at each grid point from GOES data, and second, humidity profiles are obtained from global meteorological datasets every three or six hours, where appropriate. The methodology provides the mean humidity approximately betwen 500 and $300 \mathrm{hPa}$. Thus, it is valid to obtain the PWV at sites located $5000 \mathrm{~m}$ above sea level. This methodology is applied at two high altitude sites located in the Chajnantor plateau; the APEX observatory, located at $5100 \mathrm{~m}$ and Cerro Toco, a site located at $5320 \mathrm{~m}$, where the Radiative Heating in Underexplored Bands Campaign (RHUBC-II) took place in 2009.

The PWV estimated from GOES data is a method to diagnose the amount of water vapor in the atmosphere and it could be useful in applications like site testing studies (Erasmus 2002; Erasmus \& Van Staden 2001; 2003). However, the prediction of the PWV evolution is very important for the observatories that operate at IR bands. For that reason, the simple persistence method is applied to the PWV estimated from GOES in this study to obtain short-term (12-24 h) PWV forecasts at the selected sites. Observed PWV values from radiometers at both sites are used to validate the PWV estimated from GOES and the short-term forecasts.

Section 2 describes the main features of the observations and global meteorological datasets used in the study. It also presents the methodology implemented to estimate the PWV from GOES data as well as its short-term forecasts. Section 3 shows the comparison between the PWV estimations and forecasts from GOES data and the observed PWV at both sites. Finally, Sect. 4 presents conclusions and discussions.

\section{Data and methodology}

\subsection{APEX and RHUBC-II radiometers}

The APEX is a radio telescope located at $5100 \mathrm{~m}$ above mean sea level in the Chajnantor plateau $\left(23.006^{\circ} \mathrm{S}, 67.759^{\circ} \mathrm{W}\right)$, in the Atacama Desert in the north of Chile. This telescope is an ALMA prototype antenna designed to work at submillimeter wavelengths. There is a commercial low humidity and temperature profiling (LHATPRO) microwave radiometer (Rose et al. 2005) installed at APEX that was developed by Radiometer Physics GmbH. It takes measurements at $183 \mathrm{GHz}$, which is a strong $\mathrm{H}_{2} \mathrm{O}$ emission line.

The RHUBC-II was a field experiment conducted by the US Department of Energy Atmospheric Radiation Measurement (ARM) program on Cerro Toco $\left(22.954^{\circ} \mathrm{S}, 67.769^{\circ} \mathrm{W}\right)$ in the Atacama Desert from August to October 2009 at an altitude of 5320 m (Turner \& Mlawer 2010; Turner et al. 2012). A number of instruments were deployed at the site with the main objective of measuring the radiative properties of the atmosphere in extremely dry conditions (PWV $\leq 1 \mathrm{~mm}$ ) and under temperatures and pressures that are typical of the midtroposphere. We retrieved PWV values from the GVRP ( $G$-band Vapor Radiometer Profiler), which is a 15-channel microwave radiometer that provides measurements between 170 and $183.31 \mathrm{GHz}$, but only the channels 176, 178, 180,182, and $183 \mathrm{GHz}$ were used in the physical retrieval.

Figure 1 shows the location of APEX and RHUBC-II sites in the Chajnantor plateau. We used PWV values retrieved from the radiometers at both sites to validate the PWV estimations and forecasts from GOES satellite data. 
Table 1. Description of GOES-12 channels.

\begin{tabular}{lcc}
\hline \hline Channel & Central wavelength & Resolution \\
\hline 1 Visible & $0.65(\mu \mathrm{m})$ & $1 \mathrm{~km}$ \\
\hline 2 Infrared & $3.9(\mu \mathrm{m})$ & $4 \mathrm{~km}$ \\
\hline 3 Infrared & $6.5(\mu \mathrm{m})$ & $4 \mathrm{~km}$ \\
\hline 4 Infrared & $10.7(\mu \mathrm{m})$ & $4 \mathrm{~km}$ \\
\hline 6 Infrared & $13.3(\mu \mathrm{m})$ & $8 \mathrm{~km}$ \\
\hline
\end{tabular}

\subsection{Atmospheric global data}

Data from the Global Forecast System (GFS) model and the Final Analyses (FNL) from the National Centers for Environmental Prediction (NCEP) were used to provide the temperature and humidity profiles needed to estimate the PWV from satellite data (see Sect. 2.3.3). The main characteristics of GFS and FNL data sets are the following.

\subsubsection{The GFS model}

The GFS is the global numerical weather prediction model run by NCEP four times per day $(0,6,12$ and 18 UTC) to generate forecasts up to 16 days in advance. It consists of a forecasting model and a global data assimilation system, which provides the initial meteorological state to run the model. We obtained GFS humidity and temperature profiles from 15 August to 25 October 2009 at $0.5^{\circ} \times 0.5^{\circ}$ horizontal resolution for this study every three hours. Humidity and temperature profiles are provided in pressure levels from 1000 to $900 \mathrm{hPa}$ every $25 \mathrm{hPa}$ and from $900 \mathrm{hPa}$ to $100 \mathrm{hPa}$ every $50 \mathrm{hPa}$. A complete description of the dynamical properties and the physical parametrizations included in the GFS model can be found online ${ }^{1}$.

\subsubsection{The Final analyses (FNL)}

The FNL atmospheric analysis (NCEP 2000) is created by NCEP every six hours $(0,6,12$ and 18 UTC). The FNL analysis gathers the same observations used to create the GFS operational analysis as well as other observations that were not collected in time to be included in the GFS analyses. We obtained humidity and temperature fields from the FNL at $1^{\circ} \times 1^{\circ}$ horizontal resolution for 2007 and 2009. Data are provided at 21 pressure levels from $1000 \mathrm{hPa}$ to $100 \mathrm{hPa}$ every $50 \mathrm{hPa}$.

\subsection{Estimation of PWV from GOES-12 data}

The GOES-12 takes measurements at different wavelengths, classified into five channels (Table 1). Data from channels 3 $(6.5 \mu \mathrm{m})$ and $4(10.7 \mu \mathrm{m})$ were obtained online ${ }^{2}$. The $6.5 \mu \mathrm{m}$ channel is located near the center of a strong water vapor absorption band and under clear skies is primarily sensitive to the relative humidity averaged over a deep layer centered in the upper troposphere. The $10.7 \mu \mathrm{m}$ channel is located inside an atmospheric window. Thus, it measures radiation from the surface in clear skies or cloud top radiation on cloudy days.

The GOES-12 data include the latitude, longitude, and GOES VARiable (GVAR) format count information for each pixel of $4 \mathrm{~km} \times 4 \mathrm{~km}$ horizontal resolution. We converted GVAR count values to brightness temperatures following the methodology described in Weinreb et al. (2011).

\footnotetext{
1 http://www.emc.ncep.noaa.gov/GFS/doc.php

2 http://www.class.ngdc.noaa.gov/
}

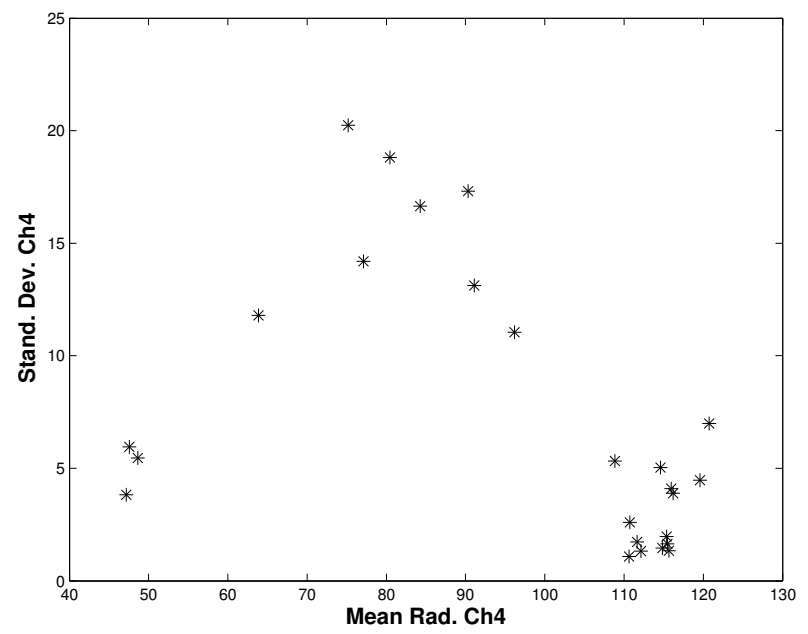

Fig. 2. Scatter plot of standard deviation ( $y$-axis) vs. mean radiances $\left(x\right.$-axis) in units of $\mathrm{mW} \mathrm{m}^{-2} \mathrm{sr}^{-1} \mathrm{~cm}$ for each $(8 \mathrm{~km})^{2}$ small matrix inside the $0.4^{\circ} \times 0.4^{\circ}$ domain for 6 January 2007 at 17:45 UTC.

\subsubsection{Cloud-clearance algorithm}

The estimation of the upper tropospheric water vapor requires that pixels are not contaminated by clouds because of their strong absorption and emission of IR radiation. For that reason, a methodology is necessary to determine cloudless radiances. The cloud clearance algorithm used by Soden \& Bretherton (1993), which is based on the spatial coherence method (SCM), was implemented in this study. The SCM uses radiation data from both the $6.5 \mu \mathrm{m}$ and $10.7 \mu \mathrm{m}$ channels in order to determine clearsky $6.5 \mu$ m radiances.

Coakley \& Bretherton (1982) introduced the SCM as an objective method to infer clear-sky IR radiation using the local spatial variability of the radiation field around a specific location. A domain of approximately $0.4^{\circ} \times 0.4^{\circ}$ is selected around the specific pixel location, which is then subdivided into several $3 \times 3(8 \mathrm{~km})^{2}$ small matrices. For each one of these small matrices, the mean and standard deviation of the $10.7 \mu \mathrm{m}$ radiance is calculated to later obtain its clear-sky value. A scatter plot of standard deviation vs mean radiance values will show an arc along mean radiance values when the domain is partially cloudy. Figure 2 shows an example of a scatter plot for 6 January 2007 at 17:45 UTC where a typical arc in radiance values can be observed. The clear-sky $10.7 \mu \mathrm{m}$ radiance value is located at the right end of the plot showing large mean radiation and low standard deviation values. On the other hand, cloudy radiances are located at the left end showing low mean radiation and low standard deviation values. Values in between will correspond to partially cloudy pixels. The clear-sky $10.7 \mu \mathrm{m}$ radiance for the site is then chosen as the largest radiance in the scatter plot among those with standard deviation values less than $5 \mathrm{~mW} \mathrm{~m}^{-2} \mathrm{sr}^{-1} \mathrm{~cm}$.

We found that a well defined arc like that shown in Fig. 2 is not always observed. This depends on the amount and type of cloud cover present in the domain. Despite this, the clearsky $10.7 \mu \mathrm{m}$ radiance is obtained using the same criteria.

The algorithm uses the fact that the mean $6.5 \mu \mathrm{m}$ radiance is approximately linear related to the mean $10.7 \mu \mathrm{m}$ radiance over the selected domain. An increase in $6.5 \mu \mathrm{m}$ radiance is accompanied by an increase in $10.7 \mu \mathrm{m}$ radiance. In the case of a fractional cloud-covered sky, the observed radiance $(R)$ in both channels $(6.5 \mu \mathrm{m}$ and $10.7 \mu \mathrm{m})$ can be estimated using the following relation from Soden \& Bretherton (1993):

$R=(1-N) R_{\mathrm{clr}}+N R_{\mathrm{cld}}$ 
where $N$ is the fractional cloud cover, $R_{\text {clr }}$ and $R_{\text {cld }}$ are the clearsky and cloudy radiance, respectively. If $N$ is eliminated using the relation 1 for both channels, the following relations are obtained:

$$
R_{6.5}=a+b R_{10.7}
$$

where

$$
b=\frac{R_{6.5 \mathrm{clr}}-R_{6.5 \mathrm{cld}}}{R_{10.7 \mathrm{clr}}-R_{10.7 \mathrm{cld}}}
$$

$a=R_{6.5 \mathrm{clr}}-b R_{10.7 \mathrm{clr}}$.

The intercept $a$ and slope $b$ are determined empirically using a linear least square fit between the observed $6.5 \mu \mathrm{m}$ and $10.7 \mu \mathrm{m}$ radiances. $R_{10.7 \mathrm{clr}}$ is determined from the SCM and then $R_{6.5 \mathrm{clr}}$ is obtained from Eqs. (3) and (4).

\subsubsection{Brightness temperature-humidity relationship}

The $6.5 \mu \mathrm{m}$ channel is located near the center of a strong water vapor absorption band. Under clear-sky conditions, it measures radiation from water vapor in a layer from roughly $300 \mathrm{hPa}$ to $600 \mathrm{hPa}$ with little contribution from lower levels. Based on analysis of GOES-7 data, Soden \& Bretherton (1993) derived a semi-empirical relationship between the relative humidity averaged over a deep layer in the upper troposphere and the $6.7 \mu \mathrm{m}$ brightness temperature in clear areas. Erasmus \& van Staden (2001) applied this empirical relation to GOES-8 data obtaining satisfactory results. The relationship is:

$\mathrm{UTH}=\frac{\exp \left(a_{2}+b_{2} \cdot T\right) \cos (\theta)}{P_{o}}$

where UTH represents the upper tropospheric humidity (values between 0 and 1), $\theta$ is the satellite viewing zenith angle, $a_{2}$ and $b_{2}$ are the least squares fit intercept and slope of the regression line as defined by the empirical relationship, and $P_{\mathrm{o}}(\mathrm{hPa})$ is a normalized pressure given by:

$P_{\mathrm{o}}(\mathrm{hPa})=\frac{p\left(T_{240 \mathrm{k}}\right)}{p 1}$

where $p(\mathrm{hPa})$ is the pressure at the level where temperature (T) equals $240 \mathrm{~K}$ and $\mathrm{p} 1$ equals $300 \mathrm{hPa}$. Soden \& Bretherton (1993) obtained the values $a_{2}=31.5$ and $b_{2}=-0.115 \mathrm{~K}^{-1}$ in their study, which are consistent with theoretical expected values from radiative transfer models. They argued that these constants are expected to have little seasonal and interannual variations. On the other hand, Erasmus \& van Staden (2001) used different values of $a_{2}$ and $b_{2}$ for each month of the year. The values of $a_{2}$ and $b_{2}$ obtained by Soden \& Bretherton (1993) were used in this study since the PWV estimated with them showed a better agreement with observations than the PWV estimated using values for $a_{2}$ and $b_{2}$ that change during the year.

In this study, Eq. (5) will be used to derive UTH values from GOES-12 brightness temperature. Although this relationship has not been validated for GOES-12 data, it is assumed that its uncertainty in the PWV estimation should be smaller than that introduced by the thermodynamic vertical profiles from numerical models and numerical calculation errors.

\subsubsection{PWV calculation}

The PWV is derived from the UTH, which is a measure of the mean relative humidity in a layer from roughly $300 \mathrm{hPa}$ to $600 \mathrm{hPa}$. The water vapor mixing ratio $\left(q_{v}\left[\mathrm{~kg} \mathrm{~kg}^{-1}\right]\right)$, i.e., the mass of water vapor per mass of dry air, can be obtained as follows:

$q_{\mathrm{v}}=\mathrm{UTH} \cdot q_{\mathrm{vs}}$

where $q_{\mathrm{vs}}$ is the saturation mixing ratio, the maximum water vapor the air can carry at a given temperature and pressure. We obtained temperature and pressure data to calculate $q_{\mathrm{vs}}$ from numerical weather models for 2007 and 2009. We selected data from 550 to $100 \mathrm{hPa}$ since APEX is located at $5100 \mathrm{~m}$ with a mean surface pressure near $550 \mathrm{hPa}$, and Cerro Toco is located at $5320 \mathrm{~m}$ with a mean surface pressure near $535 \mathrm{hPa}$ from August to September 2009.

The PWV is then calculated by:

$\mathrm{PWV}=\frac{1}{g} \int q_{\mathrm{v}} \cdot \mathrm{d} p=\frac{1}{g} \int \mathrm{UTH} \cdot q_{\mathrm{vs}} \cdot \mathrm{d} p$

where $\mathrm{d} p$ is the incremental pressure change with height in Pascals. The PWV is expressed in $\mathrm{kg} \mathrm{m}^{-2}$ or $\mathrm{mm}$ of water.

We estimated PWV values from GOES data and FNL thermodynamic profiles at $1^{\circ} \times 1^{\circ}$ horizontal resolution, every $6 \mathrm{~h}$, at the APEX site for 2007 and 2009. Thus, an extensive time period is provided to validate the estimated PWV with observations from the APEX radiometer. The results of this comparison is shown in Sect. 3.1.

We used GFS thermodynamic profiles, at a higher horizontal $\left(0.5^{\circ} \times 0.5^{\circ}\right)$ and temporal $(3 \mathrm{~h})$ resolution, together with GOES data to estimate the PWV at the APEX and RHUBC-II sites from 15 August to 25 October 2009 (the RHUBC-II period). The goals were to study the sensitivity in the PWV estimation from choosing a higher horizontal resolution profile, to analyzing how well the PWV diurnal evolution at these sites is reproduced and at what time of day the largest errors are found (see Sect. 3.2).

\subsection{Short-term PWV forecasts}

The persistence method was applied to produce short-term PWV forecasts at the two sites. The persistence method is the simplest way to produce forecasts. It assumes that the atmospheric conditions at the time of forecast will be the same as the actual conditions. For instance, if the current PWV at $12 \mathrm{UTC}$ is $0.5 \mathrm{~mm}$, then a $12 \mathrm{~h}$ forecast with the persistence method (at 24 UTC) will provide a PWV value of $0.5 \mathrm{~mm}$, since it will remain with time:

$\operatorname{PWV}(24$ UTC $)=\operatorname{PWV}(12 \mathrm{UTC})$.

This method provides the best results when the weather patterns vary little with time. Despite its simplicity, the method is used to produce short- and even long-range forecasts in different atmospheric science research areas with success (Knaff et al. 2003; Fan \& Van del Dool 2011; Guillot et al. 2012).

In the current study, $12 \mathrm{~h}$ and $24 \mathrm{~h}$ PWV forecasts were calculated from PWV estimations from GOES and GFS model data at both sites. They were compared with PWV observations to assess how useful this simple forecast method is if applied at the study sites.

The next section will show the results of the validation of the PWV estimated and forecast from GOES with observations from the APEX and RHUBC-II sites. 

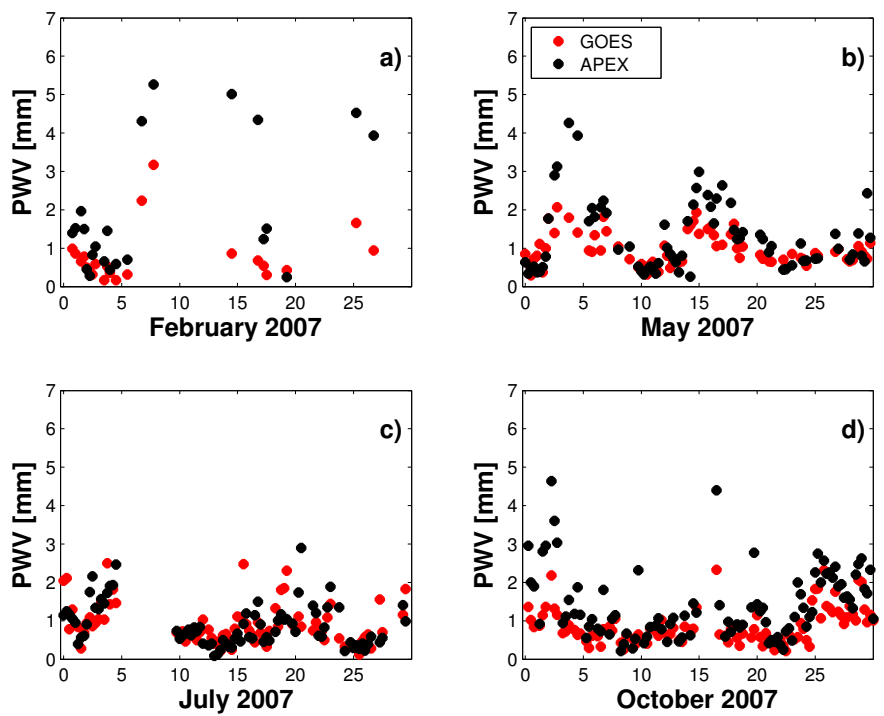

Fig. 3. Time evolution of the PWV estimated from GOES/FNL (PWV_GF, red dots) and the PWV from the APEX radiometer (PWV_A, black dots) in a) February, b) May, c) July, and d) October 2007. PWV values are shown every six hours.

\section{Results}

\subsection{PWV estimation from GOES/FNL and GOES/GFS data at the APEX site}

The analysis of the PWV estimated from GOES/FNL data for 2007 and 2009 at the APEX site is first presented in this section. After that, the PWV estimated from GOES/GFS data is shown for the period August-October 2009 at the same site.

Figures 3 and 4 show the time evolution, every six hours, of the PWV estimated from GOES/FNL (PWV_GF) and the PWV from the APEX radiometer (PWV_A) during February, May, July, and October 2007 and 2009, respectively. The plots only show times when both data were available. For that reason, few data are shown in February 2007 and February 2009 since the APEX radiometer does not take measurements during bad weather, which occurs frequently at Chajnantor in summer months.

These figures show the extremely dry conditions that are typically present at the Chajnantor plateau. Very low values predominate during a large fraction of the year, confirming the reason the ALMA project was installed at that site. Summer months experience a large number of increasing PWV episodes mainly due to the influence of strong convective storms that bring humidity and even precipitation to the region from the east (Garreaud 2009). During the rest of the year, the atmosphere is mainly affected by the passage of low-pressure systems, embedded within the westerly flow, which brings large PWV values to the region.

The PWV_GF reproduces well the time evolution of the PWV_A for most of the year, as is shown in Figs. 3b-d and 4b-d for May, July, and October 2007 and 2009, respectively. The agreement is not very good during the summer months (Figs. 3a and $4 \mathrm{a}$ ) and during periods of large PWV values that occur the rest of the year.

Low PWV values are essential for submillimeter astronomy. Values below $0.5 \mathrm{~mm}$ represent great observing conditions, while those around $1.2 \mathrm{~mm}$ represent good to decent submillimeter observations. For this reason, the discussion from now on will be focused on PWV values below $1.2 \mathrm{~mm}$.
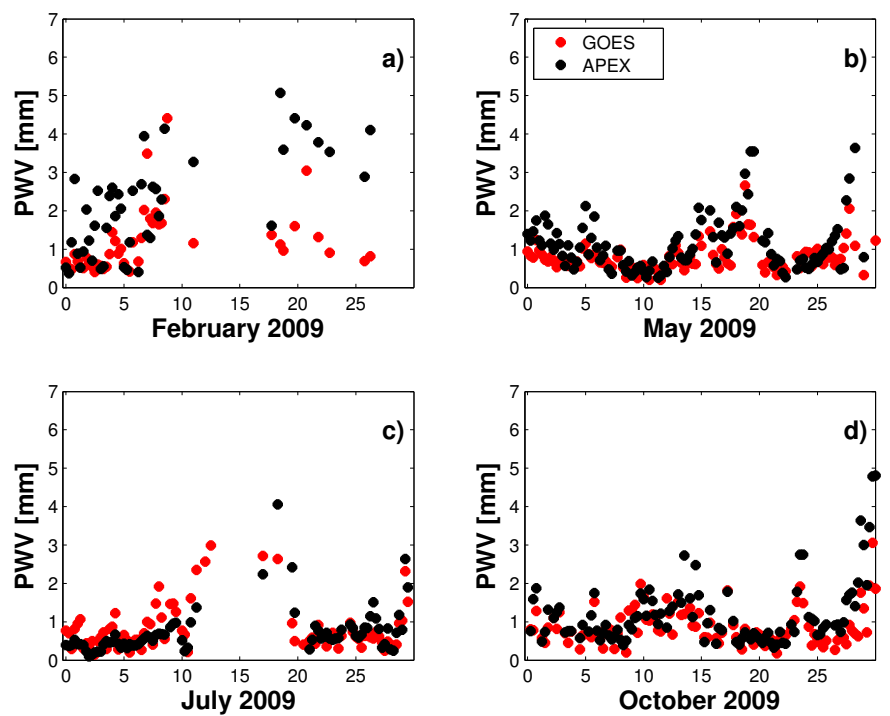

Fig. 4. Same as Fig. 3, but for a) February, b) May, c) July, and d) October 2009.

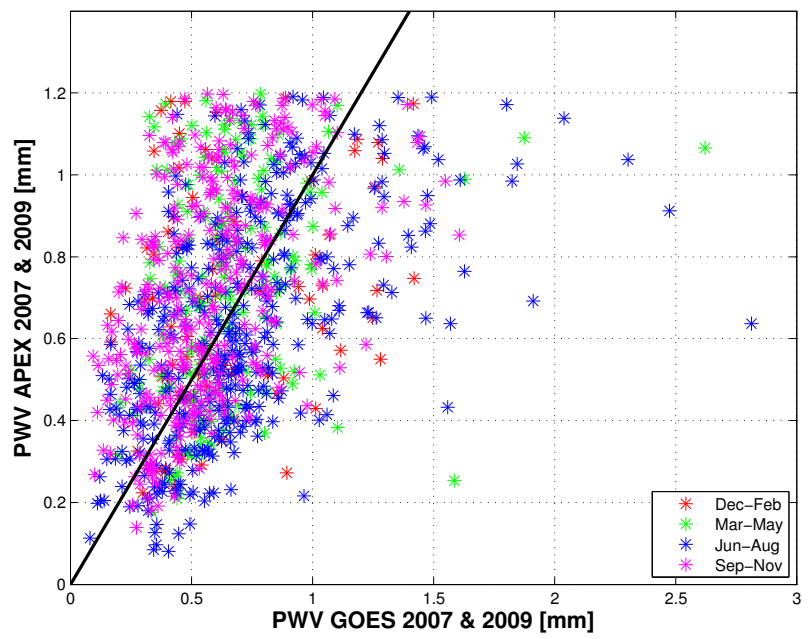

Fig. 5. Scatter plot between the PWV_GF and the PWV_A for 2007 and 2009 for the observed PWV range $0-1.2 \mathrm{~mm}$. The color stars represent PWV values for each of the four seasons of the year (southern hemisphere). See legend for details. Also shown in the plot is the line $1: 1$.

Figure 5 shows a scatter plot between the PWV_GF and the PWV_A for 2007 and 2009 for the observed PWV range $0-1.2 \mathrm{~mm}$. Data were stratified in four time periods (Dec.Feb., Mar.-May, Jun.-Aug., and Sept.-Nov.) to analyze the performance of the method during the different seasons of the year. The observed PWV at APEX seems to be mainly underestimated by GOES/FNL for values above $0.6 \mathrm{~mm}$. However, the PWV is largely overestimated at some times (although few in number), mainly during the winter. Below $0.4 \mathrm{~mm}$, the PWV_A is mainly overestimated by GOES/FNL, while no trend is evident for PWV values between $0.4 \mathrm{~mm}$ and $0.6 \mathrm{~mm}$. When the performance of the method is analyzed by season, Fig. 5 shows that the observed PWV during spring months seems to be mainly underestimated by GOES/FNL. No clear trend is shown for the other months.

The monthly mean PWV from the APEX radiometer averaged over 2007 and 2009 (Fig. 6) shows maximum values during summer and minimum values during winter. Otálora et al. (2010) also found minimum PWV values in the region during 


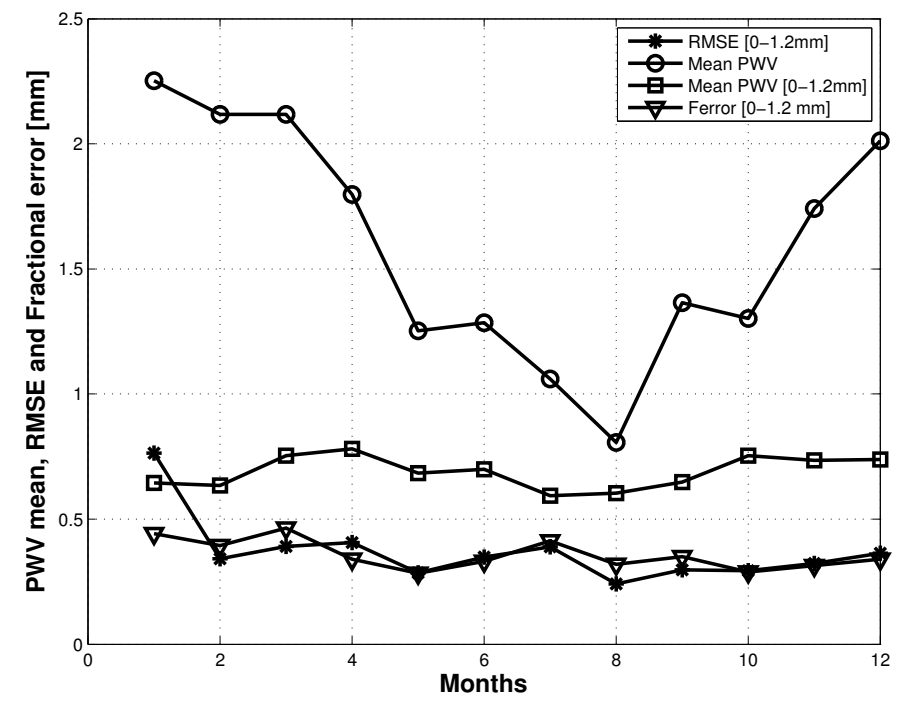

Fig. 6. Time evolution of monthly mean PWV from the APEX radiometer using all values (open circles) and only the values below $1.2 \mathrm{~mm}$ (squares) averaged over 2007 and 2009. The RMSE and the mean absolute fractional error between the PWV_GF and the PWV_A for the range $0-1.2 \mathrm{~mm}$ are shown in black stars and triangles, for both years, respectively. The RMSE and fractional error values for December are just for 2007 since GOES radiation data for that month were not available in 2009.

spring and winter months. We observed PWV differences between 2007 and 2009 (not shown). They might be associated with interannual climate variations like El Niño-southern oscillation since a different phase of the oscillation was observed in the second half of each year.

The monthly mean PWV observed in the range $0-1.2 \mathrm{~mm}$ varies little during the year, from a minimum of $0.6 \mathrm{~mm}$ in July to a maximum of $0.78 \mathrm{~mm}$ in April. The monthly root mean square error (RMSE) between GOES/FNL and observations (in the $0-1.2 \mathrm{~mm}$ range) for 2007 and 2009 is smaller than $0.4 \mathrm{~mm}$, except in January $(0.76 \mathrm{~mm})$. The latter is likely related to the occurrence of few large PWV error episodes in this summer month, as will be discussed below. The RMSE is smallest in August $(0.24 \mathrm{~mm})$ when the PWV shows the lowest value during the year.

Monthly median absolute fractional errors between the PWV_GF and the PWV_A were also calculated over the range $0-1.2 \mathrm{~mm}$. They vary between $0.29 \mathrm{~mm}$ in October and $0.46 \mathrm{~mm}$ in March. Fractional errors are close, but a bit larger than RMSEs in every months except in January, which shows a smaller value, indicating that the large RMSE shown that month could be the result of few but large PWV errors. Absolute fractional errors and RMSEs show values of $0.51 \mathrm{~mm}$ and $0.23 \mathrm{~mm}$, respectively, for the range $0-0.4 \mathrm{~mm}$ range. Absolute fractional errors and RMSEs of $0.3 \mathrm{~mm}$ and $0.36 \mathrm{~mm}$, respectively, were calculated for the range $0.4-1.2 \mathrm{~mm}$, indicating the limits of the method to estimate the PWV over these ranges at the APEX site. Based on previous results, the atmospheric transparency was calculated over a frequency interval that encompasses a strong water vapor absorption band, centered at $183 \mathrm{GHz}$, to determine the uncertainties in its calculation. The atmospheric transmissions were calculated using the Atmospheric Transmission at Microwaves (ATM) model (Pardo et al. 2001) available online ${ }^{3}$. The results are shown in Fig. 7.

\footnotetext{
3 http://almascience.eso.org/documents-and-tools/ overview/about-alma/atmosphere-model
}

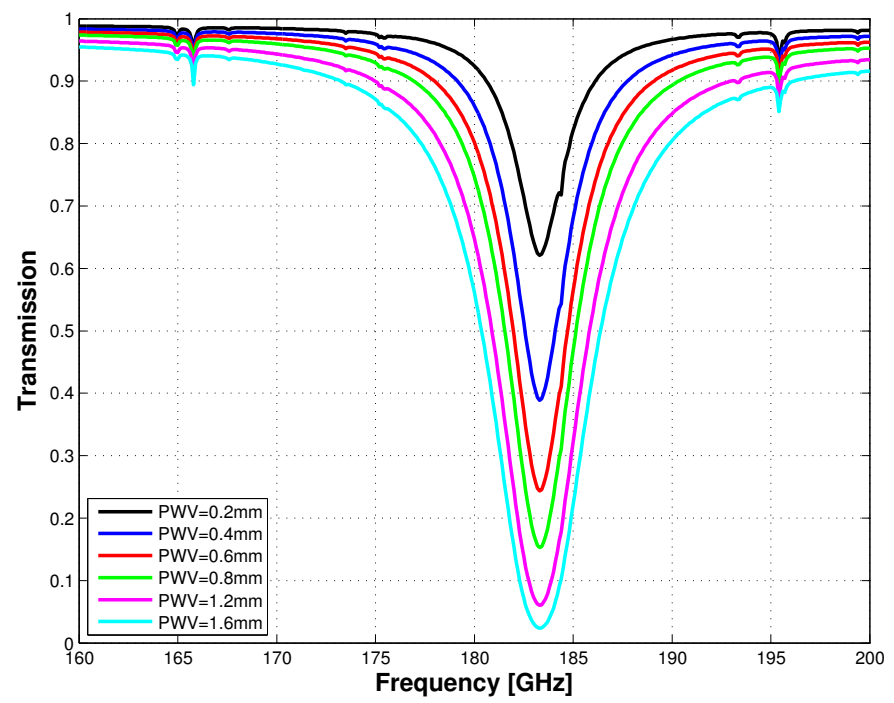

Fig. 7. Atmospheric transmissions calculated with the ATM model for six different PWV values. See legend for details.
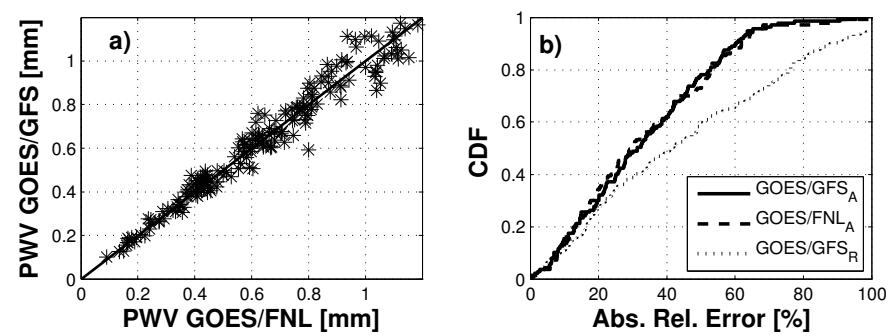

Fig. 8. a) Scatter plot of the PWV estimated from GOES/FNL versus the PWV from GOES/GFS at the APEX site from 15 August to 25 October 2009, over the range $0-1.2 \mathrm{~mm}$. The $1: 1$ line is also shown in the plot. b) CDF of PWV absolute relative errors between GOES/FNL, GOES/GFS, and observations at the APEX site (solid and dashed lines), and between GOES/GFS and observations at the RHUBC-II site (dotted line).

The PWV estimated from GOES/FNL mainly overestimates the observed PWV in the range $0-0.4 \mathrm{~mm}$ (Fig. 5) with a RMSE of $0.23 \mathrm{~mm}$. Thus, assuming a $0.2 \mathrm{~mm}$ error, the estimated atmospheric transmission uncertainties at $183 \mathrm{GHz}$ are between 0.62 and 0.4 for a PWV value of $0.2 \mathrm{~mm}$, and they are between 0.4 and 0.25 for an observed PWV of $0.4 \mathrm{~mm}$ (Fig. 7). The observed PWV above $0.6 \mathrm{~mm}$ is mainly underestimated by the GOES/FNL method, showing a RMSE value of $0.36 \mathrm{~mm}$. Therefore, assuming a $0.4 \mathrm{~mm}$ error, the transmission uncertainty could range between 0.15 and 0.35 for a PWV of $0.8 \mathrm{~mm}$ and between 0.08 and 0.15 for a PWV of $1.2 \mathrm{~mm}$, which represent good to adequate submillimeter observations.

We also calculated PWV values from GOES data and GFS thermodynamic profiles at a horizontal resolution of $0.5^{\circ} \times$ $0.5^{\circ}$ every three hours at the APEX site from 15 August to 25 October 2009, corresponding to the RHUBC-II period. They were compared with PWV values estimated from GOES/FNL thermodynamic profiles to analyze the differences between both PWV estimations due to the use of higher horizontal resolution profiles.

Figure 8a shows a scatter plot of the PWV estimated from GOES/GFS versus the PWV estimated from GOES/FNL at 0 , 6,12 , and 18 UTC for the range $0-1.2 \mathrm{~mm}$. Very small differences are shown between them, resulting in a RMSE of $0.06 \mathrm{~mm}$. The use of higher horizontal resolution profiles does not notably 


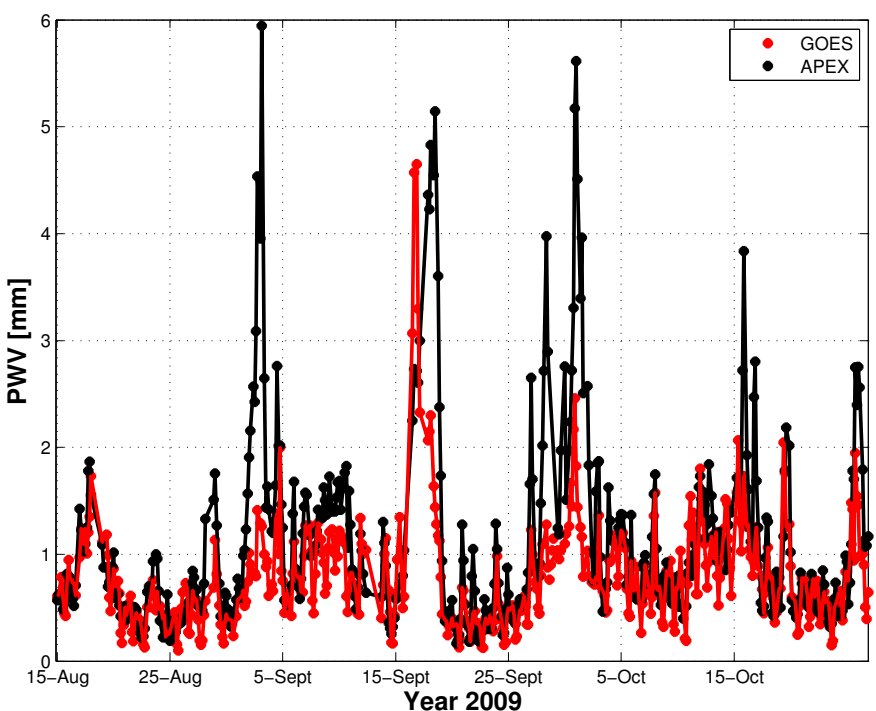

Fig. 9. Time evolution of the PWV estimated from GOES/GFS data (red dots) and the PWV from the APEX radiometer (black dots) from 15 August to 25 October 2009, every 3 hours.

improve the PWV estimation from GOES data during that period. The RMSE between GOES/FNL and APEX observations is $0.26 \mathrm{~mm}$, while that between GOES/GFS and observations is $0.25 \mathrm{~mm}$. Both estimations showed absolute relative errors below $30 \%$, in $50 \%$ of the times (Fig. 8b). However, the PWV estimated from GOES/GFS has the advantage that it can be obtained at a higher temporal resolution (every three hours), providing a better understanding of the PWV diurnal evolution, which will be discussed below. In addition, GFS analyses and forecasts are available to be downloaded earlier than FNL analyses, supporting its use to operationally estimate the PWV from GOES.

Figure 9 shows the time evolution of the PWV estimated from GOES/GFS data and the PWV from the APEX radiometer from 15 August to 25 October 2009, every three hours. Days with low PWV values (near and below $1 \mathrm{~mm}$ ) predominate during that period. They are interrupted by days with PWV values even larger than $5 \mathrm{~mm}$ mainly as a result of the passage of lowpressure systems that affect the region bringing air masses with large water vapor contents.

The estimated PWV from GOES/GFS reproduces well the APEX radiometer, particularly during days with low PWV values. The agreement is not as good on days with large PWV values, since large negative biases (GOES/GFS - APEX) are shown. Tremblin et al. (2012) also show large negative PWV biases during large PWV episodes in Chajnantor in September 2009 from Infrared Atmospheric Sounding Interferometer (IASI) polar satellite data (see Appendix A in Tremblin et al. 2012). On the other hand, Tremblin et al. (2011) found larger but positive biases between PWV values obtained from IASI satellite data and ground-based PWV observations at Dome $\mathrm{C}$, in the South Pole. The biases occurred during large PWV episodes in summer months.

A scatter plot between the PWV from GOES/GFS data and the PWV from the APEX radiometer is shown in Fig. 10a for the range $0-1.2 \mathrm{~mm}$ from 15 August to 25 October 2009. The observed PWV is mainly underestimated by the GOES/GFS data for values above $0.8 \mathrm{~mm}$. Below that value, no trend is evident. The RMSE between the APEX observations and GOES/GFS data is $0.25 \mathrm{~mm}$ for the analyzed period. The cumulative distribution function (CDF) of absolute relative errors (Fig. 8b) show that $25 \%, 50 \%$, and $75 \%$ of the PWV values estimated from
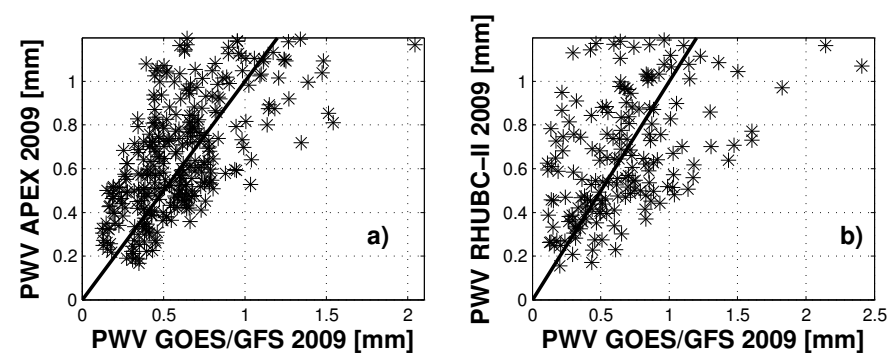

Fig. 10. Scatter plot between the PWV estimated from GOES/GFS and observations at a) APEX and b) the RHUBC-II site for the range $0-1.2 \mathrm{~mm}$ from 15 August to 25 October 2009 (the RHUBC-II period).

GOES/GFS have relative errors below $15 \%, 31 \%$, and $48 \%$, respectively. Since most of the estimated PWV during this period have absolute relative errors below $48 \%$, the related transmission uncertainty, at $183 \mathrm{GHz}$, can be estimated with the help of Fig. 7. For an observed PWV value of $0.4 \mathrm{~mm}$, the estimated atmospheric transmission uncertainty ranges between 0.62 and 0.25 , (corresponding to PWV values of $0.2 \mathrm{~mm}$ and $0.6 \mathrm{~mm}$ ). For an observed PWV value of $0.8 \mathrm{~mm}$, the estimated atmospheric transmission uncertainty ranges between 0.08 and 0.38 .

\subsection{PWV estimation from GOES/GFS at the RHUBC-II site}

Figure 10b shows a scatter plot between the PWV from GOES/GFS data and the PWV from the RHUBC-II radiometer over the range 0-1.2 mm from 15 August to 25 October 2009. Larger errors are shown at this site compared with those discussed for the APEX site. This is indicated by a RMSE of $0.35 \mathrm{~mm}$. In addition, $25 \%, 50 \%$, and $75 \%$ of the PWV values estimated from GOES/GFS have absolute relative errors below $18.6 \%, 42 \%$, and $71 \%$, respectively. Despite showing larger errors at the RHUBC-II site, $25 \%$ of the estimated PWV values show a good agreement with observations and $50 \%$ of them show a reasonably good agreement during the study period. The transmission uncertainties shown by half of the estimated PWV values at this site are similar to those shown by $75 \%$ of the PWV values estimated at the APEX site. The other half shows larger atmospheric transmission uncertainties.

\subsection{PWV diurnal evolution at APEX and RHUBC-II sites}

The mean PWV diurnal evolution at the APEX and RHUBC-II sites were calculated for values below $2 \mathrm{~mm}$ from 15 August to 25 October 2009. This allows us to analyze the PWV diurnal evolution mainly during unperturbed days, without the influence of synoptic perturbations that bring large PWV amounts to the region. Figures $11 \mathrm{a}, \mathrm{b}$ show that the observed PWV at those sites decreases from late in the afternoon to reach minimum values near sunrise. After that, the PWV increases during the morning and reaches maximum values in the afternoon. The PWV reaches larger values during the day at the RHUBC-II site than at the APEX site. Differences between both sites may be related to the different radiometers and models used to estimate the PWV and possibly to the different local meteorological conditions that affect each site. Radford (2011) also found lower PWV values (better conditions) during the nightime at the Chajnantor plateau.

The estimated PWV from GOES/GFS represents the observed PWV at both sites better during the night than during the day (Figs. 11a,b). The RMSE values smaller than $0.2 \mathrm{~mm}$ are 

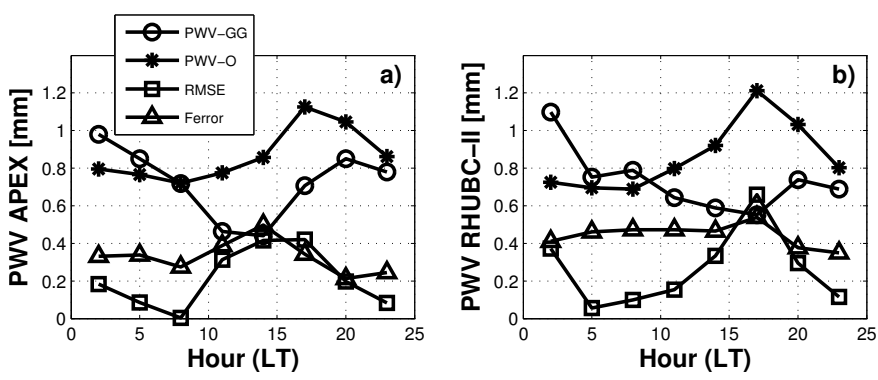

Fig. 11. Diurnal evolution of the mean PWV from GOES/GFS (circles), the mean PWV from the radiometer (stars), the RMSE (squares) and fractional error (triangles) between the PWV from GOES/GFS and observations at a) APEX and b) RHUBC-II sites calculated from 15 August to 25 October 2009.
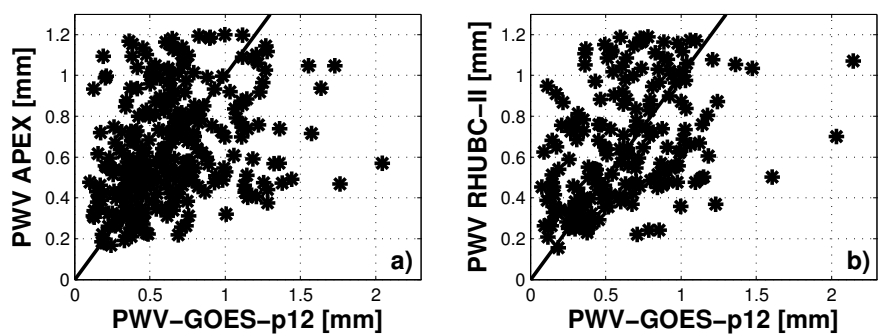

Fig. 12. Scatter plots between the $12 \mathrm{~h}$ PWV forecasts and PWV observations at a) APEX and b) RHUBC-II sites for the range $0-1.2 \mathrm{~mm}$.

shown during the night at the APEX site with mean fractional errors between 0.21 and 0.33 . The RMSE values are also smaller than $0.2 \mathrm{~mm}$ during most of the nightime at the RHUBC-II site, but fractional errors are larger (between 0.35 and 0.47). The largest PWV errors are shown during daytime, particularly during the time of maximum PWV at the RHUBC-II site. The large PWV disagreement at the RHUBC-II site is mainly due to the large bias shown during daytime since larger PWV values were observed at that site.

\subsection{Short-term PWV forecasts}

The persistence method was applied to the PWV estimated from GOES/GFS data to produce $12 \mathrm{~h}$ and $24 \mathrm{~h}$ forecasts at APEX and RHUBC-II sites from 15 August to 25 October 2009.

Figure 12 shows scatter plots between $12 \mathrm{~h}$ PWV forecasts and PWV observations at APEX and RHUBC-II sites for the range $0-1.2 \mathrm{~mm}$. The PWV forecasts do not show notable biases with observations in either of the sites. The RMSE between $12 \mathrm{~h}$ PWV forecasts and observations is $0.35 \mathrm{~mm}$ at both sites. Absolute median relative errors of $34 \%$ and $39 \%$ were shown at APEX and RHUBC-II sites, respectively. The $12 \mathrm{~h}$ forecast RMSEs over different PWV ranges (Table 2) show similar values at both sites. However, $12 \mathrm{~h}$ forecast absolute relative errors are different between the sites (Table 3). Figure 14 shows that $25 \%, 50 \%$, and $75 \%$ of $12 \mathrm{~h}$ PWV forecasts at both sites have absolute relative errors smaller than $15 \%, 38 \%$ and $60 \%$, respectively, although forecast errors at the APEX site are a bit smaller than at the RHUBC-II site. Thus, $25 \%$ of $12 \mathrm{~h}$ forecasts at both sites show a very good agreement with observations, whereas $50 \%$ of them show a reasonably good agreement with observations during the study period.

Based on the $12 \mathrm{~h}$ PWV forecast errors, the transmission uncertainties at both sites were also calculated using the ATM model. The calculated RMSE over $0-0.4 \mathrm{~mm}, 0.4-0.8$ $\mathrm{mm}$ and $0.8-1.2 \mathrm{~mm}$ can be taken as $0.25 \mathrm{~mm}, 0.35 \mathrm{~mm}$,
Table 2. RMSE between $12 \mathrm{~h}, 24 \mathrm{~h}$ PWV forecasts and observations at APEX and RHUBC-II sites over different PWV ranges.

\begin{tabular}{lcccc}
\hline \hline & \multicolumn{2}{c}{ APEX } & \multicolumn{2}{c}{ RHUBC-II } \\
\hline RMSE $[\mathrm{mm}]$ & $12 \mathrm{~h}$ & $24 \mathrm{~h}$ & $12 \mathrm{~h}$ & $24 \mathrm{~h}$ \\
\hline$[0-1.2 \mathrm{~mm}]$ & 0.35 & 0.38 & 0.35 & 0.38 \\
\hline$[0-0.4 \mathrm{~mm}]$ & 0.25 & 0.27 & 0.24 & 0.29 \\
\hline$[0.4-0.8 \mathrm{~mm}]$ & 0.35 & 0.36 & 0.36 & 0.37 \\
\hline$[0.8-1.2 \mathrm{~mm}]$ & 0.41 & 0.45 & 0.41 & 0.43 \\
\hline
\end{tabular}

Table 3. Absolute median relative errors (RE) between $12 \mathrm{~h}, 24 \mathrm{~h}$ PWV forecasts and observations at APEX and RHUBC-II sites over different PWV ranges.

\begin{tabular}{lcccc}
\hline \hline & \multicolumn{2}{c}{ APEX } & \multicolumn{2}{c}{ RHUBC-II } \\
\hline RE [\%] & $12 \mathrm{~h}$ & $24 \mathrm{~h}$ & $12 \mathrm{~h}$ & $24 \mathrm{~h}$ \\
\hline$[0-1.2 \mathrm{~mm}]$ & 34.5 & 34.2 & 39.2 & 40.3 \\
\hline$[0-0.4 \mathrm{~mm}]$ & 54.0 & 46.5 & 42.8 & 58.4 \\
\hline$[0.4-0.8 \mathrm{~mm}]$ & 33.0 & 33.3 & 47.4 & 42.7 \\
\hline$[0.8-1.2 \mathrm{~mm}]$ & 32.2 & 34.2 & 30.4 & 29.2 \\
\hline
\end{tabular}
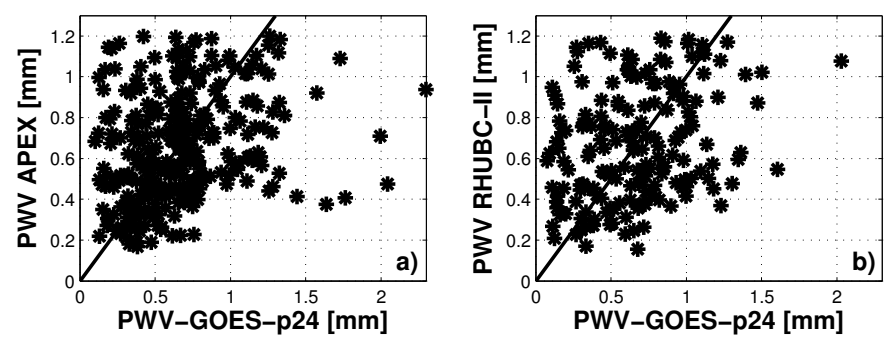

Fig. 13. Scatter plots between the $24 \mathrm{~h}$ PWV forecasts and PWV observations at a) APEX and b) RHUBC-II sites for the range 0-1.2 mm.

and $0.4 \mathrm{~mm}$, respectively, for both sites (Table 2). Thus, for observed PWV values of $0.4 \mathrm{~mm}, 0.8 \mathrm{~mm}$, and $1.0 \mathrm{~mm}$, the transmission uncertainties at both sites could range between $0.2-0.7$, $0.07-0.2$, and $0.04-0.2$, respectively. A large transmission uncertainty is estimated for PWV values below $0.4 \mathrm{~mm}$, which could make the $12 \mathrm{~h}$ PWV forecasts over this range not very useful for astronomers. However, since the estimated atmospheric transmission uncertainty for PWV values between 0.4 and $1.2 \mathrm{~mm}$ are not high, $12 \mathrm{~h}$ PWV forecasts over this range could be used with more confidence.

Scatter plots between the $24 \mathrm{~h}$ PWV forecasts and observations show no notable biases at either site (Fig. 13). The $24 \mathrm{~h}$ PWV forecasts show RMSE values of $0.38 \mathrm{~mm}$ at both sites (Table 2). However, the performance at the APEX site is better than at the RHUBC-II site (Fig. 14 and Table 3). For instance, $25 \%, 50 \%$, and $75 \%$ of the $24 \mathrm{~h}$ forecasts at the APEX site have absolute relative errors smaller than $15 \%, 34 \%$, and $62 \%$, respectivey, compared to those at the RHUBC-II site that are smaller than $21 \%, 40 \%$, and $73 \%$, respectively. Thus, $25 \%$ of the PWV forecasts at both sites show a very good agreement with observations and $50 \%$ of them show a reasonably good agreement with them. The RMSEs at $24 \mathrm{~h}$ are just a bit larger than those shown at $12 \mathrm{~h}$ at both sites. Thus, the estimated transmission uncertainties for $24 \mathrm{~h}$ PWV forecasts are similar to those discussed for $12 \mathrm{~h}$ PWV forecasts.

\section{Conclusions and discussion}

A method to estimate the PWV from GOES satellite data at high altitude sites is described in this work. This method has been 


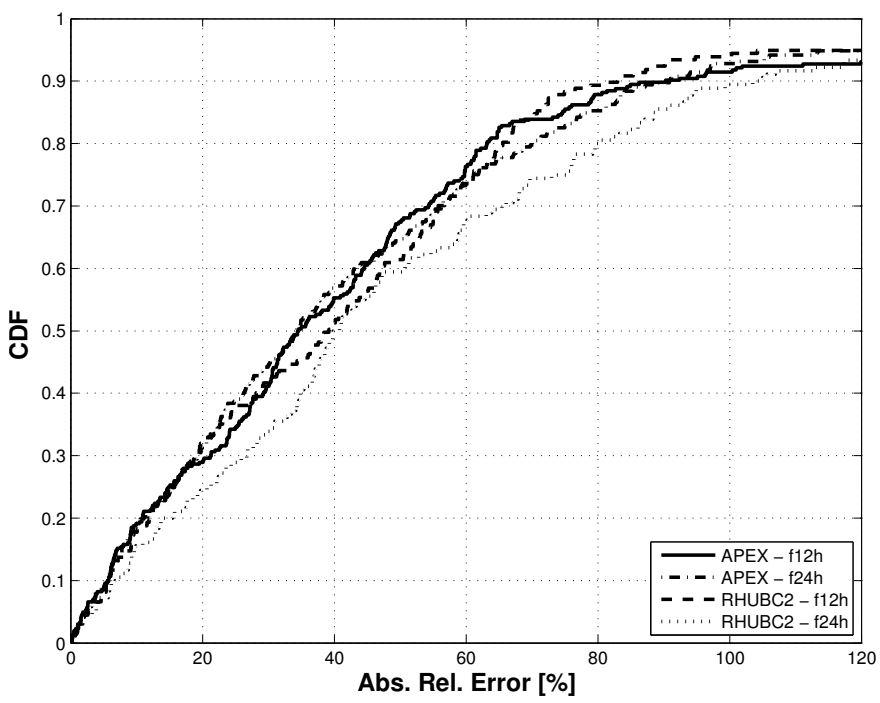

Fig. 14. CDF of absolute relative errors between 12 h, 24 h PWV forecasts from GOES/GFS and observations at APEX and RHUBC-II sites from 15 August to 25 October 2009 over the range 0-1.2 mm. See legend for details.

applied at two sites located in the Chajnantor plateau in the north of Chile: APEX, at $5100 \mathrm{~m}$ of altitude, where an ALMA prototype antenna was installed, and the RHUBC-II site, where a field experiment was conducted from August to October 2009 at $5320 \mathrm{~m}$ altitude to measure the radiative properties of the atmosphere in extremely dry conditions. The method presented here differs from a similar method that was applied in the north of Chile in that, it uses thermodynamic profiles from global meteorological models and a new cloud-clearance algorithm to obtain clear-sky brightness temperatures. A validation of the method was performed using GOES-12 satellite data over the PWV range $0-1.2 \mathrm{~mm}$ since submillimeter/millimeter astronomical observations are useful just over this range.

The PWV, estimated from GOES/FNL at APEX for 2007 and 2009, mainly overestimates the observed PWV over the range $0-0.4 \mathrm{~mm}$ whereas it mainly underestimates the PWV observations above $0.6 \mathrm{~mm}$. The RMSE between GOES/FNL and observations over the range $0-1.2 \mathrm{~mm}$ is smaller than $0.4 \mathrm{~mm}$ for the analyzed years, but a few large PWV error episodes during the summer can increase this value. The RMSE values of $0.23 \mathrm{~mm}$ and $0.36 \mathrm{~mm}$ are shown over the ranges $0-0.4 \mathrm{~mm}$ and $0.4-1.2 \mathrm{~mm}$, respectively. On the other hand, absolute relative errors of $51 \%$ and $33 \%$ are shown over these PWV ranges. The atmospheric transmission uncertainties due to the calculated absolute errors were estimated to be smaller than 0.25 over the range $0-0.4 \mathrm{~mm}$ and smaller than 0.2 over the range $0.6-1.2 \mathrm{~mm}$ for 2007 and 2009.

The PWV estimated from GOES/GFS showed a better agreement at APEX than at the RHUBC-II site. A very good agreement with observations is shown in $25 \%$ of the analyzed time (15 August to 25 October 2009), since absolute relative errors were smaller than $20 \%$. The method showed a reasonable good agreement with observations $50 \%$ of the time, with relative errors smaller than $31 \%$ and $42 \%$ at APEX and RHUBC-II sites, respectively. The estimated atmospheric transmission uncertainties at the APEX site were smaller (0.3) over the range $0.8-1.2 \mathrm{~mm}$ than over the range $0-0.4 \mathrm{~mm}(0.37)$. They were a slightly larger at the RHUBC-II site.

The use of global GFS thermodynamic profiles at $0.5^{\circ} \times$ $0.5^{\circ}$ horizontal resolution instead of FNL profiles at $1^{\circ} \times 1^{\circ}$ horizontal resolution does not show notable differences in the PWV estimation from GOES, at least during the period analyzed. However, it is recommended to use higher horizontal resolution profiles from the GFS data, since they are available every three hours and at an earlier time than the FNL data.

The analysis of the mean PWV diurnal evolution showed that the PWV estimated from GOES represents very well the observed PWV at APEX and RHUBC-II sites from August to October 2009 during night time with mean biases between -0.2 and $0.2 \mathrm{~mm}$. The largest errors are shown during daytime, particularly at the RHUBC-II site.

The analysis of $12 \mathrm{~h}$ and $24 \mathrm{~h}$ PWV forecasts at APEX and RHUBC-II sites, using the persistence method from GOES/GFS data, indicates that $25 \%$ of them show a very good agreement with observations (absolute relative errors $<20 \%$ ) whereas $50 \%$ of them show reasonably good agreement with observations (absolute relative errors $<40 \%$ ). The atmospheric transmission uncertainties using this method over the $0-0.4 \mathrm{~mm}$ ranges between 0.2 and 0.7. However, the transmission uncertainties are lower over the range $0.4-1.2 \mathrm{~mm}$. Thus, this forecast method, though very simple, yields reliable predictions of the PWV in the region at high-altitude sites. The good performance of the forecast method is due to the persistence of the atmospheric conditions that cause low PWV values during a large fraction of the year, particularly during autumn and winter months in the southern hemisphere. However, it should be used with higher confidence over the range $0.4-1.2 \mathrm{~mm}$.

In an era where many new telescope projects need a suitable place to be located, this method can be easily applied/implemented for site testing purposes as a first step to decide the location of a new astronomical site. Furthermore, it could also be used as a forecast tool, using the persistence method to provide short-term forecasts. On the other hand, global and mesoscale numerical models have provided good results in the region (Marín et al. 2013). For that reason, we are currently working with the GFS and the Weather Research and Forecasting (WRF) models, to provide accurate PWV forecasts in the Chajnantor region for a number of days in advance.

Acknowledgements. We acknowledge the ALMA-CONYCIT project 31110005 and the Fondecyt project 11121473 for supporting this research. GOES data were provided by the National Environmental Satellite, Data, and Information Service (NESDIS). GFS and FNL data were provided by the National Centers for Environmental Prediction (NCEP). We thank the anonymous reviewer for their helpful comments during the review process that largely improved the quality of the manuscript.

\section{References}

Cady-Pereira, K. E., Shephard, M. W., Turner, D. D., et al. 2008, J. Atmos. Ocean. Technol., 25, 873

Coakley, J. A., Jr., \& Bretherton, F. P. 1982, J. Geophys. Res., 87, 4917

Duan, J., Bevis, M., Fang, P., et al. 1996, J. Appl. Meteorol., 35, 830

Erasmus, D. A. 2002, The ALMA Project, European Southern Observatory, Final Report. 66814/ODG/02/6419/GWI/LET (available from: http:// www. eso.org/gen-fac/pubs/astclim/espas/radioseeing/)

Erasmus, D., \& van Staden, C. 2001, A Satellite Survey of Water Vapor and Cloud Cover in Northern Chile, Final Report, Cerro-Tololo Inter-American Observatory

Erasmus, D., \& van Staden, C. 2003, A Comparison of Satellite- Observed Cloud Cover and Water Vapor At Mauna Kea and Selected Sites In Northern Chile, The Southwestern USA and Northern Mexico, Final Report, AURA New Initiatives Office

Fan, Y., \& van den Dool, H. 2011, Weather Forecasting, 26, 355

Garreaud, R. 2009, The Andes climate and weather. Adv. Geosci, 22, 3

Guillot, E. M., Vonder Haar, T. H., Forsythe, J. M., \& Fletcher, S. J. 2012, Weather Forecasting, 27, 502 
Knaff, J. A., DeMaria, M., Sampson, C. R. \& J. M. Gross, 2003, Weather Forecasting, 18, 80

Kurlandczyk, H., \& Sarazin, M. 2007, Remote Sensing of Clouds and the Atmosphere XII, Proc. SPIE, 6745, 674507

Li, Z., Muller, J.-P., \& Cross, P. 2003, J. Geoph. Res., 108, D20, 4651

Marín, J. C., Pozo, D., Mlawer, E., Turner D., \& Curé, M. 2013, Mon. Wea. Rev., 141,3641

Miloshevich, L. M., Paukkunen, A., Vömel, H., \& Oltmans, S. J. 2004, J. Atmos. Ocean. Technol., 21, 1305

Miloshevich, L. M., Vömel, H., Whiteman, D. N., et al. 2006, J. Geophys. Res., 111, D09S 10

Miloshevich, L. M., Vömel, H., Whiteman, D. N., \& Leblanc, T. 2009, J. Geophys. Res., 114, D11305

Motell, C., Porter, J., Foster, J., \& Bevis, M. 2002, Int. J. Remote Sensing, 23, 2335

National Centers for Environmental Prediction/National Weather Service/ NOAA/US Department of Commerce 2000: NCEP FNL Operational Model Global Tropospheric Analyses, continuing from July 1999. Research Data Archive at the National Center for Atmospheric Research, Computational and Information Systems Laboratory, Boulder, CO (available online at http://dx.doi.org/10.5065/D6M043C6.)

Pardo, J. R., Cernicharo, J., \& Serabyn, E. 2001, IEEE Trans. On Antennas and Propagation, 49, 1683
Peterson, J. B., Radford, S. J. E., Ade, P. A. R., et al. 2003, PASP, 115, 805, 383 Otálora, A., Travouillon, T., Schock, M., et al. 2010, PASP, 122, 470

Radford, S. J. 2011, Rev. Mex. Astron. Astrofis., 41, 87

Rose, T., Crewell, S., Löhnert, U., \& Simmer, C. 2005, Atmos. Research, 75, 183

Soden, B. J., \& Bretherton, F. P. 1993, J. Geophys. Res., 98, 16, 669

Soden, B. J., \& Lanzante, J. R. 1996, J. Climate, 9, 1235

Soden, B. J., Turner, D., Lesht, B. M., \& Miloshevich, L. M. 2004, J. Geophys. Res. 109, D04105

Tobin, D. C., \& coauthors 2006, J. Geophys. Res., 111, D09S14

Tremblin, P., Minier, V., \& Schneider, N. 2011, A\&A, 535, A112

Tremblin, P., Schneider, N., Minier, V., Durand, G. Al., \& Urban, J. 2012, A\&A, 548, A 65

Turner, D. D., \& Mlawer, E. J. 2010, Bull. Am. Meteorol. Soc., 91, 911

Turner, D. D., Mlawer, E. J., Bianchini, G., et al. 2012, Geophys. Res. Lett., 39, L10801

Vömel, H., Selkirk, H., Miloshevich, L., et al. 2007, J. Atmos. Ocean. Technol., 24,953

Weinreb, P., Johnson, J. X., \& Han, D. 2011, NOAA Technical Memorandum. NOAA NESDIS Office of Satellite Operations, http://www.oso.noaa. gov/goes/goes-calibration/gvar-conversion.htm

Wu, P., Hamada, J.-I., \& Mori, S. 2003, J. Appl. Meteo., 42, 1107

Yang, H., Kulesa, C. A., \& Walker, C. K. 2010, PASP, 122, 890, 490 